\title{
Analysis of the invasion-metastasis mechanism in pancreatic cancer: Involvement of plasmin(ogen) cascade proteins in the invasion of pancreatic cancer cells
}

\author{
XIAODONG TAN, HIROSHI EGAMI, FUMIAKI NOZAWA, MICHIO ABE and HIDEO BABA \\ Department of Gastroenterological Surgery, Graduate School of Medical Sciences, Kumamoto University, Kumamoto, Japan
}

Received August 16, 2005; Accepted October 3, 2005

\begin{abstract}
To clarify the potential involvement of plasmin(ogen) cascade proteins in the cell dissociation and subsequent invasion of pancreatic cancer cells, Western blot analysis, immunocytochemistry, immunohistochemistry, and in vitro invasion assay were performed in the cell lines or tissue of pancreatic cancer. The strong expression of plasmin(ogen), urokinase type plasminogen activator (UPA) and UPA receptor (UPAR), and apparently weak expression of the relevant proteins were found in the conditioned medium of dissociated (PC-1.0) and non-dissociated (PC-1) pancreatic cancer cells, respectively. Furthermore, uPA-treatment significantly induced the expression of plasmin(ogen) and UPAR in the conditioned medium of non-dissociated (PC-1) pancreatic cancer cells. Moreover, the expression of plasmin(ogen) and uPAR was stronger at the invasive front than at the center of human pancreatic cancer tissue. On the other hand, plasmin-treatment induced the expression of matrix metalloproteinase-2 (MMP-2), MMP-7 and MMP-9 in PC-1 cells. Simultaneously, plasminor uPA-treatments obviously induced the dissociation of cell colonies and in vitro invasiveness in PC-1 cells. The plasmin(ogen) cascade is closely involved in the invasion of pancreatic cancer cells and, especially in its early stage, cell dissociation. Targeting the plasmin(ogen) cascade may provide a new insight into molecular target therapy based on antiinvasion and anti-metastasis for pancreatic cancer.
\end{abstract}

\section{Introduction}

Exocrine pancreatic cancer is one of the most aggressive human tumors due to its high potential of local invasion and metastasis $(1,2)$. Tumor invasion-metastasis has been known to be a complex multi-step process (3). However, thus far,

Correspondence to: Dr Xiaodong Tan, Department of Gastroenterological Surgery, Graduate School of Medical Sciences, Kumamoto University, Honjo 1-1-1, Kumamoto 860-8556, Japan E-mail: tanxd@hotmail.com

Key words: plasmin(ogen), urokinase type plasminogen activator, urokinase type plasminogen activator receptor, invasion, pancreatic cancer the cellular and molecular mechanisms of the invasionmetastasis process of pancreatic cancer are not well elucidated. In our previous studies, two hamster pancreatic cancer cell lines with a different potential of invasion-metastasis (weakly invasive cell line, PC-1, and highly invasive cell line, PC-1.0) were established $(4,5)$. In our recent investigations, activation of matrix metalloproteinase-7 (MMP-7) and the epidermal growth factor receptor (EGFR)-mediated mitogen-activated protein kinase kinase (MEK)/extracellular signal-regulated kinase (ERK) signal transduction pathway have been found to be closely associated with the high potential of cell dissociation and subsequent invasion in pancreatic cancer cells (6-8). On the other hand, two families of secreted proteases, serine proteinases [essentially the plasmin(ogen) cascade] and matrix metalloproteinases (MMPs) are important for extracellular matrix (ECM) turnover. One of the most important physiological roles of plasmin is to activate MMP-7. There are two types of plasminogen activator, urokinase type plasminogen activator (uPA) and tissue type plasminogen activator (tPA). The activation of plasminogen to plasmin by tPA or uPA binding to its $\mathrm{UPA}$ receptor (UPAR) results in progressive degradation of extracellular matrix components and basement membrane and may also lead to the activation of metalloproteinases, latent growth factors, and proteolysis of membrane glycoproteins (9). All these processes may contribute to tumor invasion-metastasis. However, reports regarding the role of the plasmin(ogen) cascade in the early stage of the invasion-metastasis process (cell dissociation) of pancreatic cancer are few. Furthermore, the relationship between the plasmin(ogen) cascade and MMPs in the early stage of invasion-metastasis of pancreatic cancer is unclear. In this study, expression of the plasmin(ogen) cascade and MMPs in pancreatic cancer was examined to clarify the involvement of the plasmin(ogen) cascade and its relationship with MMPs in the cell dissociation and subsequent invasion of pancreatic cancer cells.

\section{Materials and methods}

Cell lines and cell culture. Hamster dissociated (PC-1.0) and non-dissociated (PC-1) pancreatic cancer cell lines were used. The PC-1 cell line was established from pancreatic ductal/ductular adenocarcinomas induced by BOP in a Syrian golden hamster (4). The PC-1.0 cell line was established from a subcutaneous tumor produced after the inoculation 

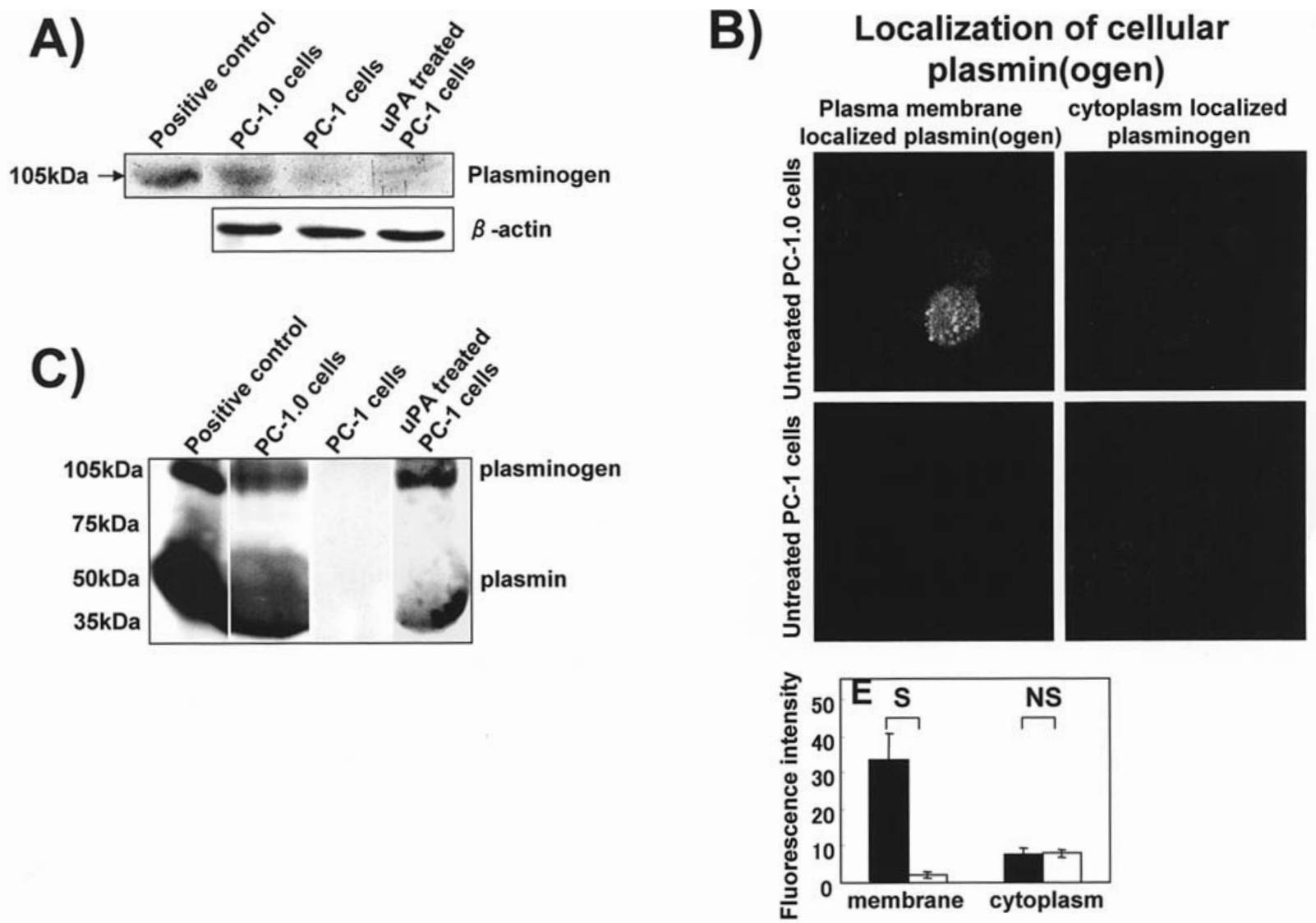

Figure 1. Plasmin(ogen) expression in pancreatic cancer cells. A), Western blotting of cellular plasminogen and plasmin proteins. Western blotting data shows that the cellular expression of plasmin(ogen) in PC-1 cells was weaker than that in PC-1.0 cells. The cellular plasmin(ogen) expression was not induced by uPA-treatment. B-actin was used as a loading control. B), localization of cellular plasmin(ogen). The constitutive expression of plasmin(ogen) on the plasma membrane of PC-1.0 cells was observed (A), but no detectable expression of plasmin(ogen) on the plasma membrane was observed in PC-1 cells (B). No detectable cytoplasm localized plasminogen expression was observed either in PC-1.0 (C) or in PC-1 cells (D). Immunofluorescent staining, original magnification $\mathrm{x} 400$. The FI of plasmin(ogen) expression is shown in (E). Black bars, PC-1.0 cells; white bars, PC-1 cells; S, significant; NS, not significant. C), Western blotting of plasminogen and plasmin proteins in conditioned medium. Constitutive expression of plasminogen and plasmin was found in the conditioned medium of PC-1.0 cells but was not detected in that of PC-1 cells. The plasminogen protein showed a single band at $105 \mathrm{kDa}$ and plasmin protein showed a wide band from 70 to $35 \mathrm{kDa}$. In addition, the plasminogen and plasmin expression was significantly induced by uPA-treatment.

of PC-1 cells (5). PC-1 cells grow as island-like colonies, whereas PC-1.0 cells exhibit a growth pattern of single cells.

The cells were incubated in RPMI-1640 (Gibco-BRL, Grand Island, NY) supplemented with $10 \%$ fetal bovine serum (Bioserum, Victoria, Australia), 100 units $/ \mathrm{ml}$ penicillin $\mathrm{G}$, and $100 \mu \mathrm{g} / \mathrm{ml}$ streptomycin at $37^{\circ} \mathrm{C}$ in a humidified atmosphere of $5 \% \mathrm{CO}_{2}$ to $95 \%$ air. The cells were serumstarved overnight before experiments.

Tissue samples. All of the tissue samples were obtained during surgery at the Department of Surgery II, Kumamoto University Hospital from October 1989 to July 2001. Specimens were from 37 pancreatic cancers. The median age of the patients with pancreatic cancer was 63.5 years (range, $35-78$ years). These patients included 14 males and 23 females. Histologically, these consisted of 13 well-differentiated, 20 moderately differentiated, and 4 poorly differentiated adenocarcinomas. All of the tissue samples were histologically examined, and the pathological diagnoses were confirmed.

Antibodies. Murine against plasminogen/plasmin antibody (American Diagnostica, CT), rabbit anti-uPA antibody (Innovative Research, Southield, MI), rabbit anti-uPAR antibody (American Diagnostica), and goat anti-MMP-2, -MMP-7, and -MMP-9 antibodies (Santa Cruz Biotechnology, Santa Cruz, CA) were used in this study. FITC labeled fluorescence secondary antibodies (Santa Cruz Biotechnology) were also used.

Preparation of cell lysate and condensation of conditioned medium. The PC-1.0 and PC-1 cells were grown in 90-mm dishes containing $10 \mathrm{ml}$ of RPMI-1640 plus $10 \%$ fetal bovine serum. After growing to subconfluence, the medium was replaced with serum-free medium and incubated for $36 \mathrm{~h}$. The PC- 1 cells were treated with or without $1 \mu \mathrm{g} / \mathrm{ml} \mathrm{uPA}$ (Innovative Research). The cell lysate and condensation of conditioned medium were prepared according to a previous study (6).

Western blot analysis. Western blotting was performed as described previously (6). In brief, samples of equivalent total protein $(20 \mu \mathrm{g})$ were run in $7.5 \%$ polyacrylamide slab gels. Human plasminogen, plasmin (Innovative Research), uPA and UPAR positive control (American Diagnostica) were used as positive control.

Immunofluorescent staining and fluorescence intensity (FI) analysis. PC-1.0 and PC-1 cells were planted on the chamber 

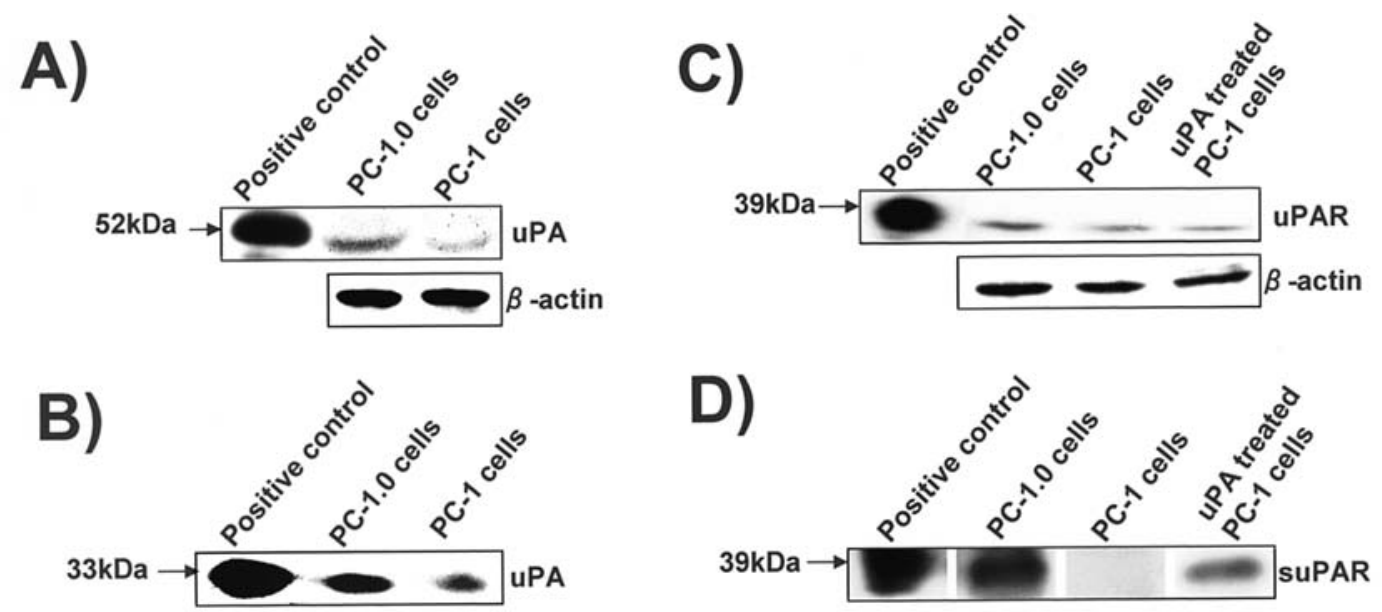

Figure 2. Western blotting of uPA and uPAR proteins in pancreatic cancer cells. (A) Cellular expression of uPA. No apparent difference was found between the cellular expression of uPA in PC-1.0 and PC-1 cells. B-actin was used as a loading control. (B) Conditioned medium localized uPA. The uPA protein in the conditioned medium of PC-1.0 cells was found to be stronger than that in the conditioned medium of PC-1 cells. (C) Cellular expression of uPAR. No difference in the cellular expression of uPAR was observed between PC-1.0 and PC-1 cells. The expression of uPAR was not induced by uPA-treatment. B-actin was used as a loading control. (D) Conditioned medium localized uPAR. The constitutive soluble uPAR (suPAR) protein was found in the conditioned medium of PC-1.0 cells, whereas no detectable band of suPAR was observed in the conditioned medium of PC-1 cells. The suPAR in the conditioned medium of PC-1 cells was significantly increased by uPA-treatment.

slides and incubated before the experiment. The PC-1 cells were treated with or without $5 \mu \mathrm{g} / \mathrm{ml}$ plasmin for $36 \mathrm{~h}$.

After incubation, immunofluorescent staining was performed as described previously (7). For examination of plasmin(ogen) expression on the plasma membrane, the cells were treated without Triton X-100. The control slides were prepared as follows: a) sections were processed without a primary antibody; b) normal goat or mouse serum and nonspecific goat or mouse $\mathrm{IgG}$ were used instead of a primary antibody.

Finally, 6 cells in the image were chosen randomly to measure the fluorescence intensity (FI) with the software Fluoview 500 (version 4.3, Olympus, Japan). The averages were used for FI analysis of the expression of plasmin(ogen), MMP-2, MMP-7, and MMP-9.

In vitro invasion assay. The in vitro invasion assay was performed using Invasion Chambers (Becton Dickinson Labware, Bedford, MA) as described previously (6). The PC-1.0 and PC-1 cells were incubated for $12 \mathrm{~h}$ at $37^{\circ} \mathrm{C}$ and PC-1 cells were pretreated with or without $5 \mu \mathrm{g} / \mathrm{ml}$ plasmin or $1 \mu \mathrm{g} / \mathrm{ml} \mathrm{uPA}$.

Immunohistochemical analysis. Immunohistochemical staining was performed using the avidin-biotin-peroxidase complex technique (Vectastain Elite ABC Kit; Vector Laboratories, Burlingame, CA) as described previously (7). The control slides were prepared as follows: a) sections were processed without a primary antibody; b) normal mouse or rabbit serum and non-specific mouse or rabbit IgG were used instead of a primary antibody.

Statistical analysis. The average FI of the expressions of plasmin(ogen), MMP-2, MMP-7, and MMP-9 in different experimental groups, as well as the numbers of pancreatic cancer cells counted in the invasion assay were examined by unpaired Student's t-test using the StatView computerized program (SAS Institute Inc., Cary, NC). A probability value $<0.05$ was considered significant.

\section{Results}

Different expressions of plasmin(ogen) cascade proteins in dissociated (PC-1.0) and non-dissociated (PC-1) pancreatic cancer cells

The plasmin(ogen) expression in $P C-1.0$ and $P C-1$ cells. The results of Western blotting showed that the cellular expression of plasmin(ogen) in PC-1.0 was stronger than that in PC-1 cells. No apparent induction of cellular expression of plasmin(ogen) in PC-1 cells was observed after uPA-treatment (Fig. 1A).

In addition, fluorescent stain images further showed that the plasma membrane localized expression of plasmin(ogen) was significantly stronger in $\mathrm{PC}-1.0$ cells $(\mathrm{FI}=33.6 \pm 7.1$, Fig. 1B-A) than in PC-1 cells $(\mathrm{FI}=2.2 \pm 0.8, \mathrm{P}<0.05$, Fig. 1B-B). However, the cytoplasm localized expression of plasminogen was weak in both PC-1.0 (FI=7.5 \pm 1.8 , Fig. 1B-C) and PC-1 cells $(\mathrm{FI}=8.0 \pm 1.1, \mathrm{P}>0.05$, Fig. 1B-D). The FI of plasmin(ogen) expression is shown in Fig. 1B-E.

In contrast to the cellular expression, the expression of plasminogen and plasmin in the conditioned medium detected by Western blotting was significantly different in PC-1.0 and PC-1 cells (Fig. 1C). A large amount of both plasminogen and plasmin proteins were detected in the conditioned medium of PC-1.0 cells, but were not detectable in that of PC-1 cells. However, uPA-treatment significantly induced the expression of plasminogen and plasmin protein in the conditioned medium of PC-1 cells.

The uPA expression in PC-1.0 and PC-1 cells. Western blotting analysis showed that the intracellular pro-uPA protein was weak, with no marked difference between PC-1.0 and PC-1 cells (Fig. 2A). On the contrary, more uPA protein was found in the conditioned medium of PC-1.0 cells than in that of PC-1 cells (Fig. 2B). 

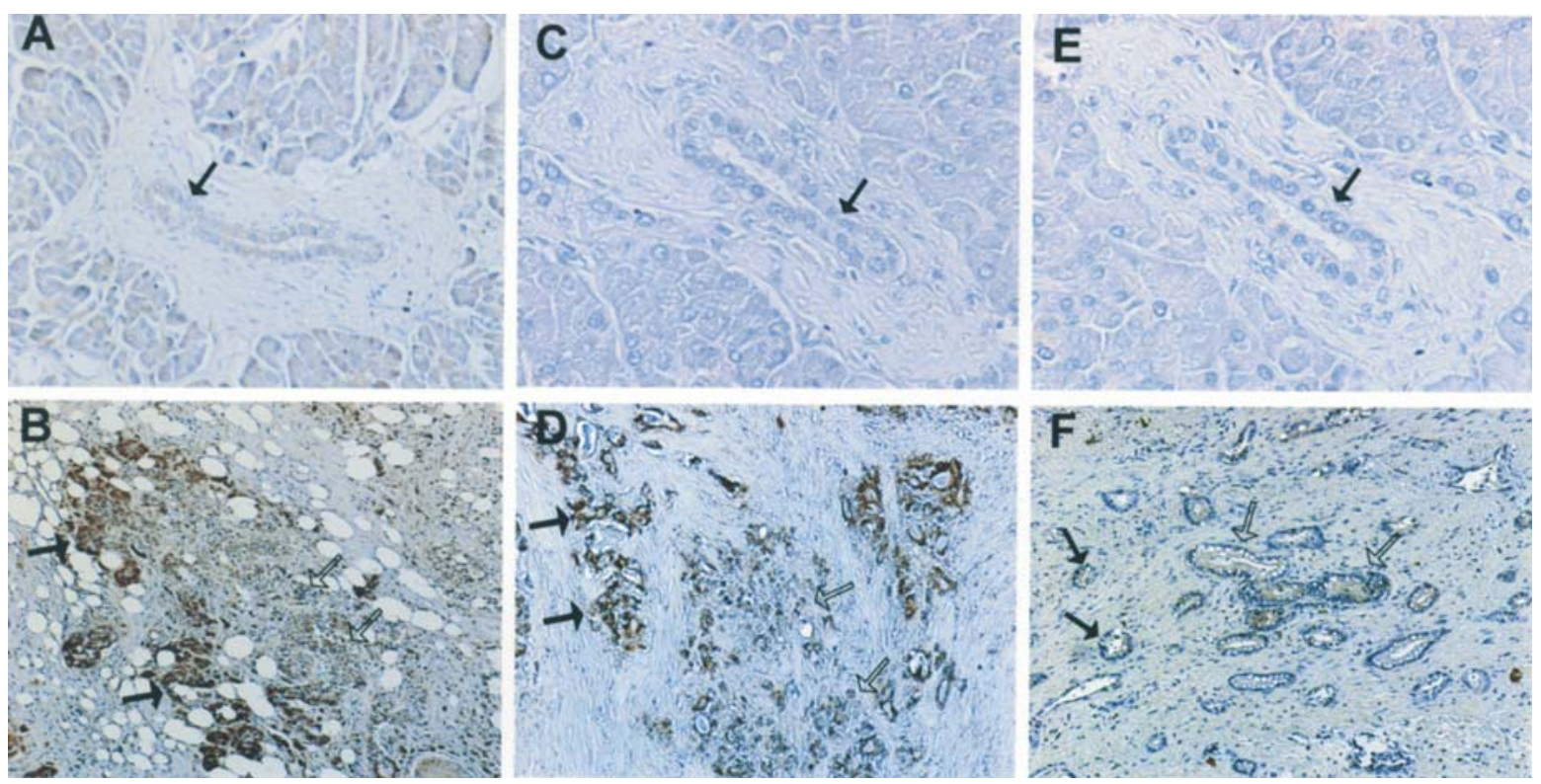

Figure 3. Expression of plasmin(ogen) cascade in human pancreatic cancer tissue. In non-malignant tissue, as shown by the black arrow, the expression of plasmin(ogen) was faint (A). The expression of uPAR (C) and uPA (E) was not detectable. On the other hand, overexpression of plasmin(ogen) (B), uPAR (D), and uPA (F) was observed in malignant tissue. Furthermore, the expression of plasmin(ogen) and uPAR was stronger at the invasive front (black arrow) than at the center (white arrow) of pancreatic cancer tissue.
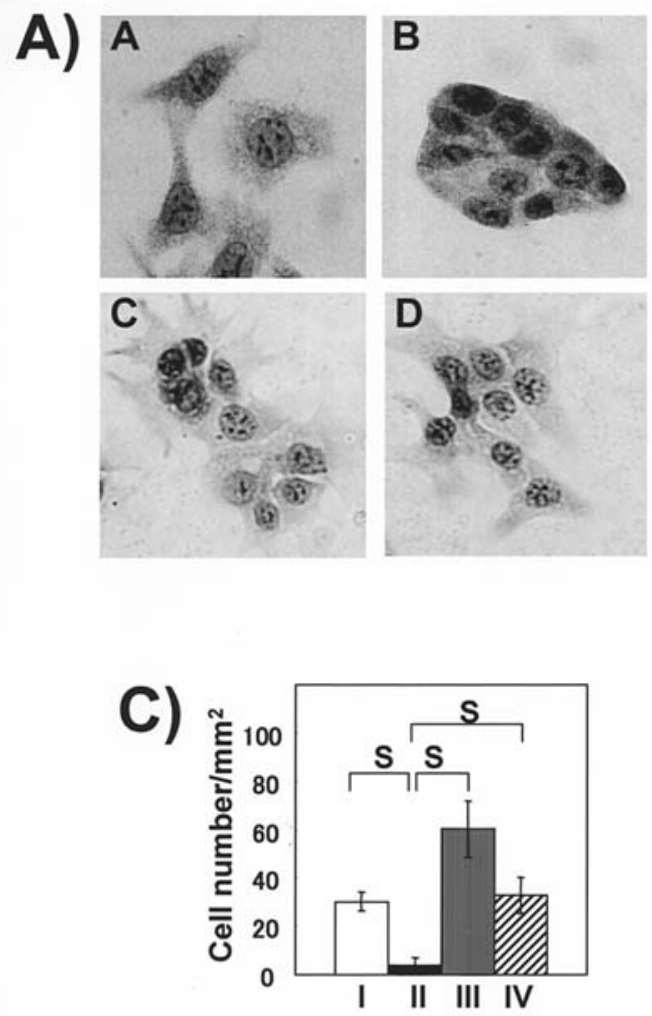

B)
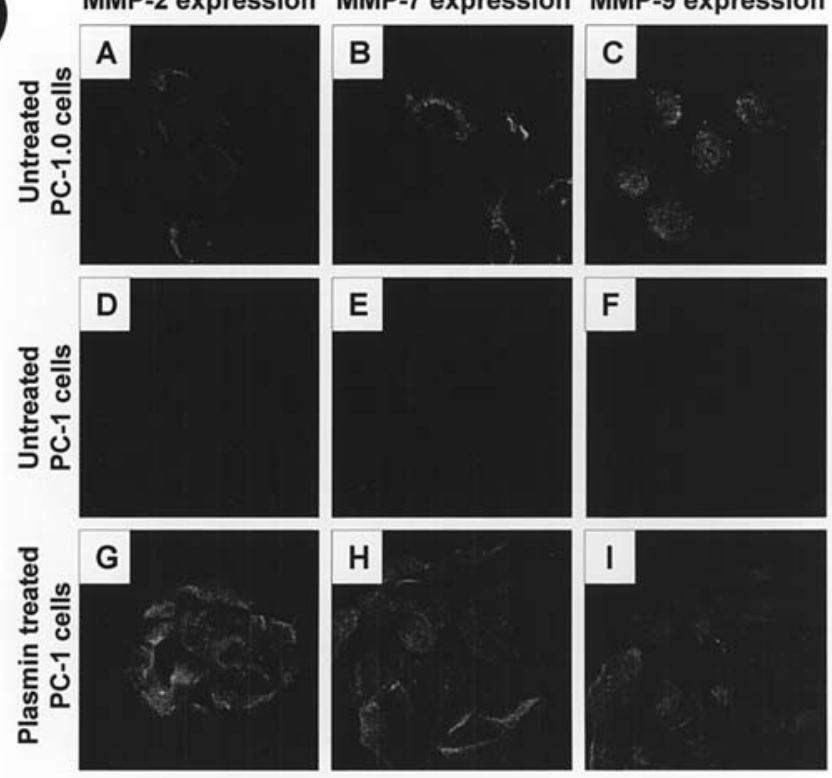

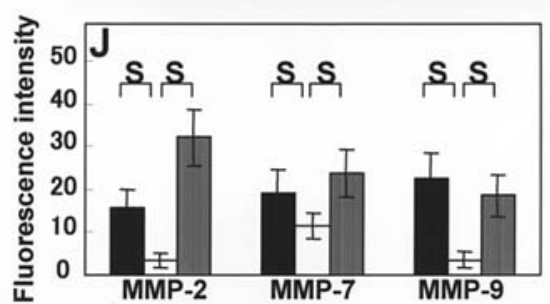

Figure 4. Effects of plasmin(ogen) cascade proteins on the cell morphology, intracellular MMP expression, as well as in vitro invasiveness of pancreatic cancer cells. A), morphological changes induced by plasmin or uPA-treatment in non-dissociated cells (PC-1). The dissociated cells (PC-1.0) were growing as single cells (A), whereas non-dissociated cells (PC-1) were growing as island-like cell colonies (B). The cell colonies of PC-1 cells were obviously dissociated and the cells elongated and formed pseudopodia by plasmin-treatment (C) and uPA-treatment (D). Papanicolaou staining, original magnification x400. B), enhanced expression of MMP-2, MMP-7, and MMP-9 by plasmin-treatment in non-dissociated cells (PC-1). Constitutive expression of MMP-2 (A), MMP-7 (B), and MMP-9 (C) was observed in PC-1.0 cells. In contrast, the expression of MMP-2 (D), MMP-7 (E), and MMP-9 (F) in PC-1 cells was faint. However, the expression of MMP-2 (G), MMP-7 (H), and MMP-9 (I) in PC-1 cells was induced by plasmin-treatment. Immunofluorescent staining, original magnification x400. The FI of MMP-2, MMP-7, and MMP-9 expression is shown in (J). Black bars, PC-1.0 cells; white bars, PC-1 cells; grey bars, plasmintreated PC-1 cells; S, significant. C), enhanced in vitro invasiveness of non-dissociated cells (PC-1) by plasmin or uPA-treatment. PC-1.0 cells present an obviously higher invasive ability than PC-1 cells. The plasmin-treatment or uPA-treatment markedly induced the invasiveness of PC-1 cells. I, PC-1.0 cells; II, PC-1 cells; III, plasmin-treated PC-1 cells; IV, uPA-treated PC-1 cells; S, significant. 
The UPAR expression in PC-1.0 and PC-1 cells. The results of Western blotting showed no apparent differences in cellular uPAR expression between PC-1.0 and PC-1 cells. Moreover, the cellular UPAR expression in PC-1 cells was not significantly induced by uPA-treatment (Fig. 2C).

However, soluble UPAR (suPAR) protein was detected in the conditioned medium of $\mathrm{PC}-1.0$ cells, whereas nearly no suPAR was detectable in the conditioned medium of PC-1 cells. In addition, the suPAR protein in the conditioned medium of PC-1 cells was significantly increased after uPA-treatment (Fig. 2D).

The expression of plasmin(ogen) cascade proteins in human pancreatic cancer tissue. In non-malignant tissue, faint immunostaining of plasmin(ogen) protein (Fig. 3A) and no immunostaining of uPAR (Fig. 3C) and uPA (Fig. 3E) proteins were observed in non-malignant pancreatic tissue. In pancreatic cancer tissue, the increased expression of plasmin(ogen) (Fig. 3B), uPAR (Fig. 3D) and uPA (Fig. 3F) was observed, both at the center (white arrow) and at the invasive front (black arrow). Furthermore, the expression of plasmin(ogen) and uPA at the invasive front was significantly stronger than that at the center of the same pancreatic cancer tissue (Fig. 3B and D, respectively).

Effects of plasmin(ogen) cascade proteins on the cell morphology, intracellular MMP expression, as well as in vitro invasiveness of pancreatic cancer cells

Induction of the dissociation of cell colonies in non-dissociated pancreatic cancer cells, PC-1, by plasmin or uPA-treatment. The dissociated pancreatic cancer cells (PC-1.0) grew as single cells (Fig. 4A-A) whereas the non-dissociated pancreatic cancer cells (PC-1) grew as island-like cell colonies (Fig. 4AB). Interestingly, the treatment with $5 \mu \mathrm{g} / \mathrm{ml}$ plasmin or $1 \mu \mathrm{g} /$ $\mathrm{ml}$ uPA for $36 \mathrm{~h}$ obviously induced the dissociation of cell colonies of PC-1 cells (Fig. 4A-C and A-D).

Induction of intracellular MMP-2, MMP-7, and MMP-9 expression in non-dissociated pancreatic cancer cells, $P C-1$, by plasmin-treatment. In dissociated cells (PC-1.0), constitutive intracellular expression of MMP-2 (FI=15.6 \pm 4.3 , Fig. 4B-A), MMP-7 (FI=19.2 \pm 5.2 , Fig. 4B-B), and MMP-9 (FI=22.6 \pm 5.6 , Fig. 4B-C) proteins was observed. However, the intracellular expression of MMP-2 (FI=3.5 $\pm 1.7, \mathrm{P}<0.05$, Fig. 4B-D), MMP-7 (FI=11.5 $\pm 2.9, \mathrm{P}<0.05$, Fig. 4B-E), and MMP-9 $(\mathrm{FI}=3.4 \pm 1.9, \mathrm{P}<0.05$, Fig. 4B-F) proteins in $\mathrm{PC}-1$ cells was weak. In contrast, the intracellular expression of MMP-2 $(\mathrm{FI}=32.0 \pm 6.7, \mathrm{P}<0.05$, Fig. 4B-G), MMP-7 (FI=23.7 \pm 5.4 , $\mathrm{P}<0.05$, Fig. 4B-H), and MMP-9 ( $\mathrm{FI}=18.6 \pm 4.9, \mathrm{P}<0.05$, Fig. 4B-I) was significantly induced in PC-1 cells by plasmintreatment. The FI of MMP-2, MMP-7, and MMP-9 expression is shown in Fig. 4B-J.

Induction of in vitro invasiveness in non-dissociated pancreatic cancer cells, $P C$-1, by plasmin- or uPA-treatment. As shown in Fig. 4C, dissociated cells (PC-1.0) exhibited a strong invasive capability (invasive cell number $=31.3 \pm 4.2$ ). Contrarily, non-dissociated pancreatic cancer cells (PC-1) showed a weak invasive capability (invasive cell number = $10.7 \pm 3.1, \mathrm{P}<0.01$ ), but the invasive capability was significantly enhanced by 12-h plasmin-treatment (invasive cell number $=$ $60.3 \pm 11.5, \mathrm{P}<0.01$ ) or uPA-treatment (invasive cell number $=$ $32.7 \pm 7.4, \mathrm{P}<0.01)$.

\section{Discussion}

Pericellular proteolysis plays a crucial role in tumor cell invasion (10). Plasmin acts both directly and indirectly (through activation of certain MMPs) to degrade proteins of the ECM and basement membrane, thereby facilitating ECM degradation, tumor proliferation, invasion and metastasis $(11,12)$. In the current study, plasmin(ogen) was distinctly expressed in pancreatic cancer cells which have apparently different dissociation status and invasion abilities. A strong plasmin(ogen) expression was detected at the plasma membrane and in the conditioned medium of dissociated cells, which were growing as single cells and had a high invasion ability. On the contrary, the plasmin(ogen) expression was not detectable either at the plasma membrane or in the conditioned medium of non-dissociated cells, which were growing as island-like cell colonies and had a weak invasion ability. Moreover, plasmin-treatment significantly induced the cell dissociation and in vitro invasion ability of nondissociated cells. In addition, the plasmin(ogen) protein was overexpressed in human pancreatic cancer tissue and a stronger plasmin(ogen) expression was observed at the invasive front than at the center of pancreatic cancer tissue. Collectively, the current results demonstrate that plasmin(ogen) is closely involved in the invasion-metastasis process of pancreatic cancer cells, especially in the process of cell dissociation, which is its first important step. The differently expressed plasmin(ogen) at the plasma membrane and in the conditioned medium rather than in the cytoplasm of pancreatic cancer cells may imply that the interaction of plasmin(ogen) with other molecules is essential for the activation and functioning of plasmin(ogen).

On the other hand, the basement membrane is composed mainly of type IV collagen, laminin, heparin sulfate proteoglycans, and entactin (13). Gelatinase A (MMP-2) and gelatinase B (MMP-9), and their balance in cancer cells is thought to be related to the invasiveness of the cells (14). In addition, MMP-7 positivity was reported to be significantly correlated with the extent of tumor invasion, lymph node and distant metastasis in pancreatic carcinoma (15). In the current study, the simultaneous induction of cell dissociation and expression of MMP-2, MMP-7, and MMP-9 by plasmintreatment were observed in non-dissociated cells, PC-1. These results indicate that interactions between the plasmin(ogen) cascade and MMPs may cooperate in achieving extracellular matrix degradation. Furthermore, several active MMPs can activate other MMPs, thus representing a positive-feedback mechanism $(16,17)$. As a result, cell dissociation and an enhanced invasiveness of pancreatic cancer cells are induced.

uPA, another important molecule in the plasmin(ogen) cascade, was also found to be differently expressed in the conditioned medium of dissociated and non-dissociated pancreatic cancer cells. uPA-treatment induced the plasmin(ogen) expression, cell dissociation, as well as invasiveness of non-dissociated cells. However, tPA, another plasminogen activator, showed no difference in expression 
between the cytoplasm or conditioned medium of dissociated and non-dissociated cells in the preliminary experiment (data not shown). Although tPA can also convert plasminogen to plasmin, it is mainly reported to be involved in fibrinolysis (18). These results suggest that it may be uPA which serves as a plasminogen activator in the cell dissociation and subsequent invasion-metastasis process of pancreatic cancer cells.

Compared with cellular localized UPAR, the UPAR present in body fluids (soluble UPAR, suPAR) was reported to be a more important prognostic marker $(19,20)$. Although no obvious difference in uPAR expression was observed between the cytoplasm of dissociated and non-dissociated cells, more suPAR protein was found in the conditioned medium of dissociated cells than in the conditioned medium of nondissociated cells in this study. Furthermore, the suPAR in the conditioned medium of non-dissociated cells was markedly increased by uPA-treatment. The UPAR protein was also found to be overexpressed in human pancreatic cancer tissue and the expression at the invasive front was stronger than that at the center of the pancreatic cancer tissue. Hence, all these data may indicate that UPAR is also one of the important molecules involved in the invasion-metastasis process of pancreatic cancer. However, because no difference in expression of uPA was found between the invasive front and the center of pancreatic cancer tissue, the different expression patterns of UPAR and UPA in pancreatic cancer tissue imply that, besides binding to UPA, uPAR may play an independent role in the invasion-metastasis of pancreatic cancer.

In summary, the plasmin(ogen) cascade is closely involved in the cell dissociation and subsequent invasion of pancreatic cancer cells. Production, secretion, and activation of plasminogen, rather than its activators UPA and tPA, possibly serves as a rate-limited step in the cell dissociation and subsequent invasion of pancreatic cancer. Targeting the invasive properties of pancreatic cancer, such as the plasmin(ogen) cascade, may provide a new therapeutic strategy for the anti-tumor therapy of pancreatic cancer.

\section{References}

1. Jaffee EM, Hruban RH, Canto M and Kern SE: Focus on pancreas cancer. Cancer Cell 2: 25-28, 2002.

2. Real FX: A 'catastrophic hypothesis' for pancreas cancer progression. Gastroenterology 124: 1958-1964, 2003.

3. Torimura T, Ueno T, Kin M, et al: Autocrine motility factor enhances hepatoma cell invasion across the basement membrane through activation of $\beta 1$ integrins. Hepatology 34: 62-71, 2001.

4. Egami H, Takiyama Y, Cano M, Houser WH and Pour PM: Establishment of hamster pancreatic ductal carcinoma cell line (PC-1) producing blood group-related antigens. Carcinogenesis 10: 861-869, 1989.
5. Egami H, Tomioka T, Tempero M, Kay D and Pour PM: Development of intrapancreatic transplantable model of pancreatic duct adenocarcinoma in Syrian golden hamsters. Am J Pathol 138: 557-561, 1991.

6. Tan X, Egami H, Ishikawa S, et al: Involvement of matrix metalloproteinase-7 in invasion-metastasis through induction of cell dissociation in pancreatic cancer. Int J Oncol 26: 1283-1289, 2005.

7. Tan X, Egami H, Kamohara H, et al: Involvement of the mitogen-activated protein kinase kinase 2 in the induction of cell dissociation in pancreatic cancer. Int J Oncol 24: 65-73, 2004.

8. Tan X, Egami H, Abe M, Nozawa F, Hirota M and Ogawa M: Involvement of matrix metalloproteinase-7 in invasion of pancreatic cancer cells through activation of EGFR-mediated MEK/ERK signal transduction pathway. J Clin Pathol (In press).

9. De Clerck YA, Imren S, Montgomery AM, Mueller BM, Reisfeld RA and Laug WE: Proteases and protease inhibitors in tumor progression. Adv Exp Med Biol 425: 89-97, 1997.

10. Blasi F: Urokinase and urokinase receptor: a paracrine/autocrine system regulating cell migration and invasiveness. Bioessays 15: 105-111, 1993.

11. Andreasen PA, Kjoller L, Christensen L and Duffy MJ: The urokinase-type plasminogen activator system in cancer metastasis: a review. Int J Cancer 72: 1-22, 1997.

12. Schmitt M, Harbeck N, Thomssen C, et al: Clinical impact of the plasminogen activation system in tumor invasion and metastasis: prognostic relevance and target for therapy. Thromb Haemost 78: 285-296, 1997.

13. Kawada M and Umezawa K: Suppression of in vitro invasion of human fibrosarcoma cells by a leupeptin analogue inhibiting the urokinase-plasmin system. Biochem Biophys Res Commun 209: 25-30, 1995 .

14. Wilhelm SM, Collier IE, Marmer BL, Eisen AZ, Grant GA and Goldberg GI: SV40-transformed human lung fibroblasts secrete a 92-kDa type IV collagenase which is identical to that secreted by normal human macrophages. J Biol Chem 264: 17213-17221, 1989.

15. Yamamoto H, Itoh F, Iku S, et al: Expression of matrix metalloproteinases and tissue inhibitors of metalloproteinases in human pancreatic adenocarcinomas: clinicopathologic and prognostic significance of matrilysin expression. J Clin Oncol 19: 1118-1127, 2001.

16. Ogata Y, Enghild JJ and Nagase H: Matrix metalloproteinase 3 (stromelysin) activates the precursor for the human matrix metalloproteinase 9. J Biol Chem 267: 3581-3584, 1992.

17. Imai K, Yokohama Y, Nakanishi I, et al: Matrix metalloproteinase 7 (matrilysin) from human rectal carcinoma cells: activation of the precursor, interaction with other matrix metalloproteinases and enzymic properties. J Biol Chem 270: 6691-6697, 1995.

18. Collen D and Lijnen HR: Basic and clinical aspects of fibrinolysis and thrombolysis. Blood 78: 3114-3124, 1991.

19. Sier CF, Stephens R, Bizik J, et al: The level of urokinase-type plasminogen activator receptor is increased in serum of ovarian cancer patients. Cancer Res 58: 1843-1849, 1998.

20. Miyake H, Hara I, Yamanaka K, Gohji K, Arakawa S and Kamidono S: Evaluation of serum levels of urokinase plasminogen activator and its receptor is associated with disease progression and prognosis in patients with prostate cancer. Prostate 39: 123-129, 1999. 